OPEN ACCESS

Edited by:

Jeanette M. Norton,

Utah State University, USA

Reviewed by:

Anthony Yannarell,

University of Illinois

at Urbana-Champaign, USA

Hongchen Jiang,

Miami University, USA

*Correspondence:

C. Ryan Penton

crpenton@asu.edu

Specialty section:

This article was submitted to

Terrestrial Microbiology,

a section of the journal

Frontiers in Microbiology

Received: 01 April 2016 Accepted: 16 May 2016

Published: 02 June 2016

Citation:

Penton CR, Gupta WSR, Yu J and

Tiedje JM (2016) Size Matters: Assessing Optimum Soil Sample Size for Fungal and Bacterial Community

Structure Analyses Using High

Throughput Sequencing

of rRNA Gene Amplicons.

Front. Microbiol. 7:824.

doi: 10.3389/fmicb.2016.00824

\section{Size Matters: Assessing Optimum Soil Sample Size for Fungal and Bacterial Community Structure Analyses Using High Throughput Sequencing of rRNA Gene Amplicons}

\author{
C. Ryan Penton ${ }^{1,2 *}$, Vadakattu V. S. R. Gupta ${ }^{3}$, Julian Yu ${ }^{1}$ and James M. Tiedje ${ }^{4}$ \\ ${ }^{1}$ Faculty of Science and Mathematics, College of Integrative Sciences and Arts, Arizona State University, Mesa, AZ, USA, \\ ${ }^{2}$ Arizona State University Applied and Functional Microbiomics Institute, Arizona State University, Mesa, AZ, USA, ${ }^{3}$ CSIRO \\ Agriculture, Glen Osmond, SA, Australia, ${ }^{4}$ Center for Microbial Ecology, Michigan State University, East Lansing, MI, USA
}

We examined the effect of different soil sample sizes obtained from an agricultural field, under a single cropping system uniform in soil properties and aboveground crop responses, on bacterial and fungal community structure and microbial diversity indices. DNA extracted from soil sample sizes of $0.25,1,5$, and $10 \mathrm{~g}$ using MoBIO kits and from 10 and $100 \mathrm{~g}$ sizes using a bead-beating method (SARDI) were used as templates for high-throughput sequencing of $16 \mathrm{~S}$ and $28 \mathrm{~S}$ rRNA gene amplicons for bacteria and fungi, respectively, on the Illumina MiSeq and Roche 454 platforms. Sample size significantly affected overall bacterial and fungal community structure, replicate dispersion and the number of operational taxonomic units (OTUs) retrieved. Richness, evenness and diversity were also significantly affected. The largest diversity estimates were always associated with the $10 \mathrm{~g} \mathrm{MoBIO}$ extractions with a corresponding reduction in replicate dispersion. For the fungal data, smaller MoBIO extractions identified more unclassified Eukaryota incertae sedis and unclassified glomeromycota while the SARDI method retrieved more abundant OTUs containing unclassified Pleosporales and the fungal genera Alternaria and Cercophora. Overall, these findings indicate that a $10 \mathrm{~g}$ soil DNA extraction is most suitable for both soil bacterial and fungal communities for retrieving optimal diversity while still capturing rarer taxa in concert with decreasing replicate variation.

Keywords: DNA extraction, fungal community, microbial ecology, sample size, microbial diversity

\section{INTRODUCTION}

The complex structural and spatial physico-chemical heterogeneity of soils likely influences microbial community structure, particularly over varying spatial scales. Microbial populations can be preferentially localized in microhabitats, e.g., rhizosphere, detritusphere, drillosphere, aggregatosphere, pores, organic matter coatings, etc. that provide suitable habitat requirements (Hattori, 1988; Bailey et al., 2013; Vos et al., 2013). Spatial heterogeneity due to carbon and nutrient availability and redox potential gradients also promote diversity by providing specific niches and creating ecological opportunities (Rainey and Travisano, 1998; Gupta and Germida, 2015). Given this complexity, soil microbial biology was often treated as a "black box" (Tiedje et al., 1999) but, with the advent 
of molecular methods, that box began to open to better reveal the players and their activities as influenced by management and environmental attributes.

Sample size, along with replication, sampling design, DNA extraction, and molecular analyses (currently amplicon sequencing methods), affect measures of community structure including alpha and beta diversity, dispersion, and hence comparisons among treatments and among experiments. Given the spatial heterogeneity of soil, sample size should be large enough to encompass all the significant microhabitats of the ecological unit under study so that larger drivers of biological structure can be discerned.

While sampling strategies for high-throughput amplicon sequencing studies often focus on the number of replicates taken in order to increase the power of statistical analyses, they often ignore the size of the soil sample used for DNA extraction. Prior studies have given some general insight into the influence of sample size on community structure results. Ellinsøe and Johnsen (2002), using denaturing gradient gel electrophoresis (DGGE), found larger variations in community structure among replicates with small sample sizes ( 0.01 and $0.1 \mathrm{~g}$ ) versus larger samples (1.0 and $10 \mathrm{~g}$ ). They concluded that small soil samples harbor bacterial communities that are missed in larger soil samples. Ranjard et al. (2003), using automated ribosomal intergenic spacer analysis (ARISA) on soil sample sizes from 0.125 to $4 \mathrm{~g}$, found that bacterial community structures were similar for all sample sizes, but fungal communities had higher replicate variation, particularly in small sample sizes. They suggested that small soil samples more accurately reflect the fungal composition than that observed in larger soil samples. The central conclusion drawn from this study was that large soil samples are most suitable for the description of the overall soil community but large numbers of small samples are more appropriate for a determination of local microbial diversity.

Archaeal community structure assessed by DGGE was more similar among $10 \mathrm{~g}$ replicates than 0.1 and $1 \mathrm{~g}$ extraction sizes (Nicol et al., 2003). Their general conclusion agreed with Ranjard et al. (2003) in that rare members of the archaeal community would likely not be observed using large samples and that an extensive microsampling approach was necessary to assess the rare components that are present only in microenvironments. Sample size also affects ecofunctional gene analysis; Stres et al. (2004) found increasing convergence of RFLP profiles of nos $Z$ (nitrous oxide reductase) as sample sizes reached 1 to $3 \mathrm{~g}$ samples, which then allowed distinctions among sites. Kang and Mills (2006), using DGGE and soil extraction sizes ranging from 0.01 to $10 \mathrm{~g}$ from a native temperate tallgrass meadow, found that replicate dispersion was lowest for the bacterial community in the 0.25 to $10 \mathrm{~g}$ samples while the fungal communities were clustered in the 0.1 to $0.25 \mathrm{~g}$ samples. They concluded that $0.25 \mathrm{~g}$ was optimal for the assessment of both bacterial and fungal communities from a single DNA extraction. These studies show that small samples detect more rare members but resulted in more variation among replication and hence less ability to distinguish among treatments or conditions. No studies have evaluated effects of sample sizes, ranging from $0.25 \mathrm{~g}$ to $100 \mathrm{~g}$, using the much higher through resolution and sampling depth provided by high throughput sequencing. However, Song et al. (2015) did find that with increasing sample size there was an increase (although non-significant) in the average OTU richness from fungal amplicon (ITS) sequence data for prairie and forest soils in USA in soil sample sizes ranging from $0.25 \mathrm{~g}$ to $10 \mathrm{~g}$.

The aim of this study was to evaluate the effect of microscale heterogeneity by utilizing a range of soil sample sizes 0.25 to $100 \mathrm{~g} \mathrm{-} \mathrm{for} \mathrm{DNA} \mathrm{extraction} \mathrm{on} \mathrm{both} \mathrm{bacterial} \mathrm{and}$ fungal community structure measures as determined by highthroughput amplicon sequencing of the bacterial $16 \mathrm{~S}$ and the fungal $28 \mathrm{~S}$ rRNA genes. An optimum sample size was found for overall community structure, replicate dispersion, within replicate similarity and variations in diversity indices among soil extraction sizes and methodologies. Pairwise comparisons of extractions were used to determine the presence of large discrepancies in the relative abundances of specific taxa due to sample size.

\section{MATERIALS AND METHODS}

\section{Sample Description and DNA Extraction}

Four GPS locations in one agricultural field located at Avon in South Australia (S34 13.981, E138 18.586) were sampled during the non-crop season in March, 2012. At each location a $15 \mathrm{~m} \times 12 \mathrm{~m}$ area was marked as a field replicate (see Supplementary Figure S1 for sampling design). The soil type is Luvic Calcisol and sandy to sandy loam in texture (Lithocalcic Calcarosol) (Northcote et al., 1975). Soil physiochemical properties were: clay $17 \%$, sand $51 \%$, silt $32 \%$, organic C $1.6 \%$, total $\mathrm{N} 0.15 \%$, and $\mathrm{pH}$ (water) 8.3. The site was cropped in cereals (wheat, barley, or oats) for at least 5 years. Two independent collections of three $40 \mathrm{~mm}$ diameter cores, at randomly selected points $(\sim 490$ g soil each), were taken at each of the four GPS locations within the field (Supplementary Figure S1). In order to reduce large-scale heterogeneity while preserving aggregate structure and retaining microscale heterogeneity, each group of three cores was gently mixed yielding a composited sample representing each of the four field replicate locations. Large un-decomposed plant material and stones were removed. The sandy loam texture of the soil does not allow formation of large aggregates or clods that requires sieving of soil. Subsamples of different sizes $(0.25 \mathrm{~g}, 0.5 \mathrm{~g}, 1.0 \mathrm{~g}, 10 \mathrm{~g}$, and $100 \mathrm{~g})$ were taken from each of the two composited samples consisting of three cores, for a total of four samples for each of the four field locations, resulting in 16 samples per sample size for MoBIO extraction (Supplementary Figure S1). For SARDI DNA extraction, one sample from each composite was taken, resulting in 8 samples for SARDI $10 \mathrm{~g}$ and $100 \mathrm{~g}$ extractions. Soil samples were immediately placed on ice in a cooler, transported to the laboratory and stored at $-20^{\circ} \mathrm{C}$ until lyophilized for SARDI extraction, or until shipped on dry ice to Michigan State University for DNA extraction as follows. Genomic DNA was extracted from $0.25 \mathrm{~g}$ soil sub-samples using the MoBIO PowerSoil DNA Isolation Kit. 1.0 g, 5 g, and $10 \mathrm{~g}$ soil samples were extracted using the MoBIO PowerMax Soil DNA Isolation Kit following manufacturer's instructions. The additional $10 \mathrm{~g}$ and 
$100 \mathrm{~g}$ soil samples remaining in Australia were extracted by the South Australian Research and Development Institute (SARDI, Adelaide, AU, USA) Root Disease Testing Service (Ophel-Keller et al., 2008). SARDI utilizes a bead-beating method and has been demonstrated to be an effective method for quantifying plant roots (Haling et al., 2011; Huang et al., 2013) and soil fungi (Simpson et al., 2011; Bithell et al., 2013). In total there were 16-0.25 g, 16-1.0 g, 16-5 g, 8-10 g (MoBIO), 8-10 g (SARDI), and 8-100 g (SARDI) soil DNA extractions used as templates for PCR amplification at the Center for Microbial Ecology at Michigan State University.

\section{$28 S$ and 16S rRNA Gene Amplification}

Fungal 28S rRNA gene amplicons were generated using primers LR3/LR0R ${ }^{1}$ (Liu et al., 2012) according to previously published protocols (Penton et al., 2013, 2014). Quadruplicate amplification replicates were pooled and gel purified using the Qiagen Gel Purification Kit following band excision then further purified using the Qiagen PCR Purification Kit. Following adapter ligation, amplicons were sequenced by the Utah State University CIB Genomics Core Lab on the 454 Titanium platform.

Bacterial 16S rRNA genes were amplified using the dual index paired-end approach for the Illumina MiSeq platform (Kozich et al., 2013). Briefly, each primer consisted of an Illumina adapter, an 8-nt index sequence, 10-nt pad sequences, a 2-nt linker and the 16S V4 primer sequence forward (CCTACGGGAGGCAGCAG) or reverse (GGACTACHVGGGTWTCTAAT). Amplification was performed on a 96-well plate using AccuPrime Pfx SuperMix reagents and library clean-up and normalization was performed using the Invitrogen SequalPrep Plate Normalization Kit. The library QC was performed using a KAPA Biosystems qPCR kit and by obtaining a bioanalyzer trace using the Agilent Technologies HS DNA kit. Sequencing was done at Michigan State University's Research and Technology Support Facility.

\section{Sequence Processing and Statistics}

Raw 28S rRNA gene sequences were processed for minimum length (400 bp), quality $(Q>20)$, primer match and barcode sorting using the RDP pyrosequencing pipeline. Chimeras were identified and removed using UCHIME (Edgar et al., 2011) in de-novo mode and the remaining sequences were randomly resampled to 4,300 sequences per sample using MOTHUR (Schloss et al., 2009). Three samples were discarded that did not meet the minimum resampling depth. The remaining 266,600 sequences were aligned then clustered at 5\% nucleotide dissimilarity and representative sequences generated for each OTU using RDP tools hosted on the Michigan State University High Performance Computing Center servers ${ }^{2}$. The RDP Fungal Classifier ${ }^{3}$ based on training set 11 was used for classification of each cluster representative sequence.

Bacterial 16S rRNA gene amplicons were sequenced on the Illumina MiSeq platform ( $2 \mathrm{bp} \times 250 \mathrm{bp}$ paired end reads). Raw reads were assembled using a modified PandaSeq (Cole

\footnotetext{
${ }^{1}$ http://www.biology.duke.edu/fungi/mycolab/primers.htm

${ }^{2}$ http://icer.msu.edu/hpcc

${ }^{3}$ http://rdp.cme.msu.edu
}

et al., 2014) with a minimum overlap of $50 \mathrm{bp}$, minimum and maximum lengths of 220 and 280, respectively, and a minimum Q score of 28 as determined by defined community analysis using RDP tools (Fish et al., 2013). All computation was performed on the MSU High Performance Computing Center servers. UCHIME (Edgar et al., 2011) was used to identify and remove chimeras followed by resampling at 23,000 sequences per sample using MOTHUR (Schloss et al., 2009), alignment then clustering at 3\% nucleotide dissimilarity. Representative sequences were classified using the RDP Classifier with training set 9 at $80 \%$ confidence.

Raw cluster abundances were Hellinger transformed and a Bray-Curtis dissimilarity matrix $(+1)$ was constructed, statistical analyses performed and diversity estimates calculated using PRIMER-E (Clarke and Gorley, 2006). Statistical analyses were based on four replicates from each of the four field GPS locations $(n=16)$, except for SARDI $10 \mathrm{~g}$ and $100 \mathrm{~g}$ extractions $(n=8)$. Cluster analysis was performed with the Similarity Profile analysis (SIMPROF) test (Clarke et al., 2008). Significant differences in community structure were tested using Permutational Multivariate Analysis of Variance (PERMANOVA) (Anderson, 2001) and Analysis of Similarity (ANOSIM) (Clarke, 1993). Sample replicate dispersion was tested by Permutational Analysis of Multivariate Dispersions (PERMDISP) (Anderson et al., 2006) and a test for Multivariate Dispersion (MVDISP). ANOVA statistics for Shannon diversity (H'), Pielou's Evenness (J), Margalef's Richness (d) and the number of individuals $(\mathrm{N})$ were performed using Minitab 16 (Minitab Inc, USA). Sequences were deposited in the European Nucleotide Archive 4 under study PRJEB8081 with accession numbers ERS632772-632841 and ERS671660-ERS671724.

\section{RESULTS}

\section{Sequencing}

A total of 591,120 fungal 28S rRNA gene sequences were retrieved after initial processing for quality, length, and matches to the forward primer sequence; $2.8 \%$ of all sequences were identified as chimeras and removed prior to re-sampling. Clustering at $5 \%$ nucleotide dissimilarity on 4,300 sequences per sample yielded 23,431 clusters of which 14,352 were singletons or doubletons. For the bacterial $16 \mathrm{~S}$ rRNA genes, a total of 5,501,355 raw paired end reads produced from 70 samples yielded 4,130,058 assembled and quality-filtered reads. A total of $1.3 \%$ of filtered reads were identified as chimeras and removed. Clustering at 3\% nucleotide dissimilarity on 23,000 sequences per sample yielded 18,355 OTUs of which 4736 were singletons and 1840 were doubletons.

\section{Community Differences with Sample Size and Extraction Method}

For both the bacterial and fungal communities, Margalef's richness (d, ANOVA, 28S: $F=11.25, P<0.001,16 \mathrm{~S}: F=18.42$, $P<0.001$ ), Pielou's Evenness (J', 28S: $F=6.3, P<0.001,16 \mathrm{~S}$ :

${ }^{4}$ http://www.ebi.ac.uk/ena/ 
$F=10.81, P<0.001)$, Shannon Diversity (H', 28S: $F=9.1$, $P<0.001,16 \mathrm{~S}: F=15.65, P<0.001)$ and the number of individuals $(\mathrm{N}, 28 \mathrm{~S}: F=7.9, P<0.001,16 \mathrm{~S}: F=15.58$, $P<0.001)$ were significantly different among extraction sizes in both datasets, with the highest values always associated with the $10 \mathrm{~g}$ MoBIO extraction (Table 1).

Significant differences in fungal and bacterial community composition were identified from PERMANOVA analysis among soil sample sizes (28S: $F=2.18, P=0.001,16 \mathrm{~S}: F=2.79$, $P=0.001$ ) and among replicates (28S: $F=1.41, P=0.001$, 16S: $F=1.27, P=0.013)$ but not with the interaction terms of size $\times$ replicate $(28 \mathrm{~S}: F=0.86, P>0.10,16 \mathrm{~S}$ : $F=0.94, P>0.10)$. Permutational dispersion (PERMDISP) revealed significant overall differences in sample dispersion among extraction sizes (28S: $F=43.6, P=0.001,16 \mathrm{~S}$ : $F=14.47$, $P=0.001)$ with dispersion values decreasing with increasing sample extraction size (28S: ANOVA, $F=43.60, P=0.001$, 16S: $F=14.5, P<0.001$ ) (Table 2). Decreasing multivariate dispersion indices (MVDISP) with increasing extraction size for both $28 \mathrm{~S}$ and $16 \mathrm{~S}$ datasets was also found. PERMANOVAbased similarities of within replicate groups for $16 \mathrm{~S}$ data also increased with sample size from $34.5 \%$ in $0.25 \mathrm{~g}$ to $48.8 \%$ in $100 \mathrm{~g}$ (Table 2). For the fungal data the within-group similarities increased from $62.5 \%$ in $0.25 \mathrm{~g}$ to $67.7 \%$ in $100 \mathrm{~g}$, although there was a decrease associated with the 1 and $5 \mathrm{~g}$ samples. Dispersion among the sub-replicates was calculated using PERMDISP and the four values for each extraction size were averaged (Dmean Rep, Table 2). These dispersions showed that similarity among sub-samples increased as extraction size increased. The number of total OTUs retrieved was significantly different among sample sizes (ANOVA, 28S: $F=10.91, P<0.001,16 \mathrm{~S}: F=17.63$, $P<0.001$ ) as were the non-singleton/doubleton OTUs (ANOVA, 28S: $F=5.08, P=0.001,16 \mathrm{~S}: F=14.47, P<0.001)$.

For both $28 \mathrm{~S}$ and $16 \mathrm{~S}$ the largest number of OTUs was associated with the $10 \mathrm{~g}$ SARDI and $10 \mathrm{~g}$ MoBIO extractions, respectively (Table 3 ). After the removal of singleton-doubleton OTUs, the bacterial data again showed that $10 \mathrm{~g}$ MoBIO resulted in the highest number of OTUs (total and unique sequences), though by a small margin over $10 \mathrm{~g}$ SARDI. For the fungal data, the highest number of non-singleton-doubleton OTUs originated from the $1 \mathrm{~g}$ sample, with the $10 \mathrm{~g}$ MoBIO a close second. Fungal OTU-based rarefaction data (Figure 1A) showed smaller replicate variance in the $10 \mathrm{~g}, 10 \mathrm{~g}$ SARDI and $100 \mathrm{~g}$ SARDI sequence data. The $10 \mathrm{~g}$ extractions consistently showed higher coverage, especially compared to the $0.25 \mathrm{~g}$ and $1 \mathrm{~g}$ samples. Bacterial OTU-based rarefaction data (Figure 1B) illustrated the same trend with the additional observation that the $0.25 \mathrm{~g}, 1 \mathrm{~g}$, and $5 \mathrm{~g}$ extractions especially showed a trend toward earlier saturation.

In total, $31.3 \%$ of the bacterial (Figure 2) and $69.5 \%$ of the fungal (Figure 3) sequences were shared among all extraction sizes, including both extraction methods. For bacteria, the most

TABLE 1 | Diversity indices for 28S and 16S rRNA genes according to sample extraction size for Margalef's richness (d), Pielou's evenness ( $\mathrm{J}$ '), Shannon Diversity ( $\left.\mathrm{H}^{\prime}\right)$, and the overall number of individuals (N) with ANOVA grouping with Tukey's test at $95 \%$ confidence shown by superscript letters.

\begin{tabular}{|c|c|c|c|c|c|c|c|c|}
\hline \multirow[b]{2}{*}{ Size } & \multicolumn{4}{|c|}{$28 S$} & \multicolumn{4}{|c|}{$16 S$} \\
\hline & $d$ & $J^{\prime}$ & $H^{\prime}$ & $N$ & $d$ & J' & $H^{\prime}$ & $N$ \\
\hline $0.25 \mathrm{~g}$ & $239.6^{\mathrm{B}}$ & $0.981^{\mathrm{C}}$ & $7.09^{\mathrm{B}}$ & $313^{C}$ & $648.3^{\mathrm{B}}$ & $0.976^{\mathrm{B}}$ & $8.10^{\mathrm{B}}$ & $506.3^{\mathrm{BC}}$ \\
\hline $1 \mathrm{~g}$ & $241.1^{\mathrm{B}}$ & $0.983^{\mathrm{BC}}$ & $7.12^{\mathrm{B}}$ & $322^{\mathrm{BC}}$ & $544.2^{\mathrm{C}}$ & $0.977^{\mathrm{B}}$ & $7.92^{C}$ & $469.1^{C}$ \\
\hline $5 \mathrm{~g}$ & $269.3^{A}$ & $0.985^{\mathrm{AB}}$ & $7.25^{\mathrm{A}}$ & $345^{\mathrm{AB}}$ & $660.0^{\mathrm{B}}$ & $0.977^{\mathrm{B}}$ & $8.13^{\mathrm{B}}$ & $514.4^{\mathrm{B}}$ \\
\hline $10 \mathrm{~g}$ & $285.5^{A}$ & $0.987^{A}$ & $7.33^{A}$ & $365^{A}$ & $779.6^{A}$ & $0.979^{A}$ & $8.33^{A}$ & $572.7^{A}$ \\
\hline $10 \mathrm{~g}$ (SARDI) & $238.2^{\mathrm{B}}$ & $0.982^{\mathrm{BC}}$ & $7.10^{\mathrm{B}}$ & $317^{\mathrm{BC}}$ & $766.3^{A}$ & $0.979^{A}$ & $8.31^{\mathrm{A}}$ & $566.3^{A}$ \\
\hline $100 \mathrm{~g}$ (SARDI) & $237.1^{\mathrm{B}}$ & $0.982^{\mathrm{BC}}$ & $7.09^{B}$ & $317^{\mathrm{BC}}$ & $715.5^{\mathrm{AB}}$ & $0.977^{A B}$ & $8.22^{\mathrm{AB}}$ & $537.8^{\mathrm{AB}}$ \\
\hline
\end{tabular}

The highest values in each column are bolded for reference.

TABLE 2 | 28 S and 16S rRNA gene results from the permutational dispersion (PERMDISP) test showing dispersion means (Dmean) and standard errors (SE) for the extraction size groups.

\begin{tabular}{|c|c|c|c|c|c|c|c|c|c|c|}
\hline \multirow[b]{2}{*}{ Extraction Size } & \multicolumn{5}{|c|}{$28 S$} & \multicolumn{5}{|c|}{$16 S$} \\
\hline & Dmean & SE & SIM & MVD & $\begin{array}{c}\text { Dmean } \\
\text { Rep }\end{array}$ & Dmean & SE & SIM & MVD & $\begin{array}{c}\text { Dmean } \\
\text { Rep }\end{array}$ \\
\hline $0.25 \mathrm{~g}$ & $44.66^{A}$ & 0.70 & $34.5 \%$ & 1.21 & 38.9 & $25.62^{\mathrm{BC}}$ & 0.48 & $62.5 \%$ & 1.03 & 22.3 \\
\hline $1 \mathrm{~g}$ & $44.40^{\mathrm{A}}$ & 0.91 & $34.4 \%$ & 1.24 & 36.3 & $29.12^{A}$ & 1.55 & $55.4 \%$ & 1.69 & 23.5 \\
\hline $5 g$ & $44.68^{A}$ & 0.36 & $34.3 \%$ & 1.27 & 37.4 & $27.70^{\mathrm{AB}}$ & 0.86 & $59.4 \%$ & 1.34 & 23.9 \\
\hline $10 \mathrm{~g}$ & $37.79^{\mathrm{B}}$ & 0.45 & $37.3 \%$ & 0.34 & 28.4 & $23.44^{C D}$ & 0.27 & $64.6 \%$ & 0.72 & 17.7 \\
\hline $\log (\mathrm{S})$ & $38.43^{\mathrm{B}}$ & 0.59 & $42.3 \%$ & 0.36 & 29.5 & $22.25^{D}$ & 0.35 & $66.4 \%$ & 0.36 & 16.7 \\
\hline $100 \mathrm{~g}(\mathrm{~S})$ & $33.54^{C}$ & 0.26 & $48.8 \%$ & 0.07 & 25.6 & $\mathbf{2 1 . 4 0 ^ { D }}$ & 0.39 & $67.6 \%$ & 0.19 & 15.9 \\
\hline
\end{tabular}

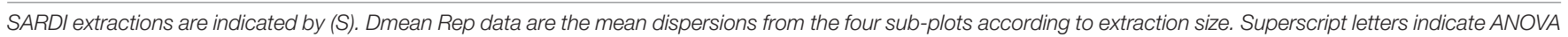

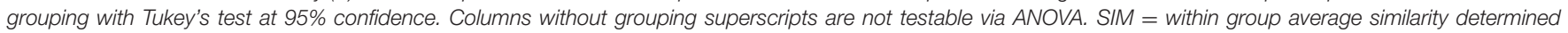

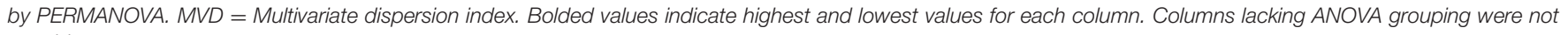
testable. 
TABLE 3 | Average number of total rRNA OTUs and of the non-singleton or doubleton (Non-S/D) OTUs retrieved from each sample size.

\begin{tabular}{|c|c|c|c|c|}
\hline \multirow[b]{2}{*}{ Extraction Size } & \multicolumn{2}{|c|}{ Total OTUs } & \multicolumn{2}{|c|}{ Non-S/D OTUs } \\
\hline & $28 S$ & $16 S$ & $28 S$ & $16 S$ \\
\hline $0.25 \mathrm{~g}$ & $1379^{A}$ & $3960^{\mathrm{A}}$ & $319^{A}$ & $1722^{\mathrm{A}}$ \\
\hline $1 \mathrm{~g}$ & $1394^{\mathrm{A}}$ & $3314^{\mathrm{B}}$ & $366^{\mathrm{B}}$ & $1769^{\mathrm{AB}}$ \\
\hline $5 g$ & $1576^{\mathrm{B}}$ & $4016^{A}$ & $339^{A B C}$ & $1764^{\mathrm{A}}$ \\
\hline $10 \mathrm{~g}$ & $1394^{\mathrm{B}}$ & $4701^{C}$ & $360^{\mathrm{BC}}$ & $1869^{\mathrm{B}}$ \\
\hline 10g (SARDI) & $1685^{\mathrm{A}}$ & $4636^{C}$ & $330^{A C}$ & $1868^{\mathrm{B}}$ \\
\hline 100g (SARDI) & $1366^{\mathrm{A}}$ & $4358^{A C}$ & $334^{\mathrm{ABC}}$ & $1785^{\mathrm{AB}}$ \\
\hline
\end{tabular}

ANOVA grouping with Tukey's test at 95\% confidence are denoted by superscript letters. The largest and smallest values in each column are bolded for reference.

abundant unique sequences for any one extraction generally belonged to the Proteobacteria, Planctomycetes, Acidobacteria, Actinobacteria, and Firmicutes, though they comprised less than
$0.05 \%$ of all sequences. For the fungi, unique sequences were somewhat less rare, though they did not exceed $1.3 \%$ of the total. The most abundant unique fungal sequences belonged to the Chytridiomycetes, Sordariomycetes, Dothideomycetes, and Blastocladiomycetes. Among these, the Dothideomycetes appear to be more frequently common than unique in any one sizeextraction method sample. In addition, the proportion of unique OTUs to unique sequences in any one sample indicates that these unique sequences were relegated to low abundance OTUs. The $0.25 \mathrm{~g}$ and $10 \mathrm{~g}$ MoBio samples shared $98.0 \%$ of bacterial and $83.8 \%$ of fungal sequences contained within 60.0 and $30.5 \%$ of the total OTUs, respectively.

Extraction methods were explicitly compared using the MoBIO $10 \mathrm{~g}$ and SARDI $10 \mathrm{~g}$ samples. Overall community structure was significantly different between extraction methods (PERMANOVA, monte-carlo, 28S: $P=0.002,16 \mathrm{~S}: P=0.001$ ). Furthermore, all diversity estimates were significantly larger in

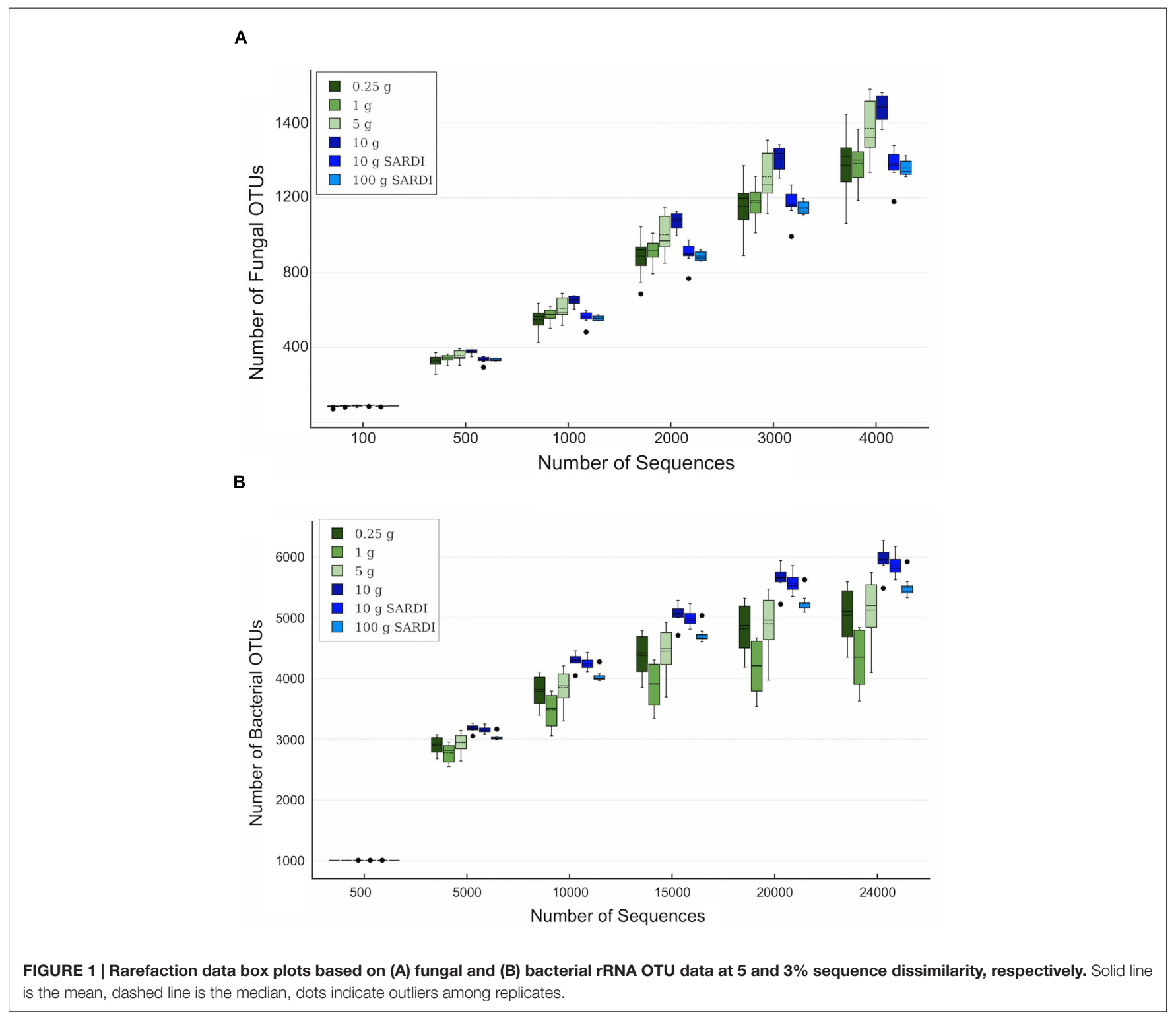




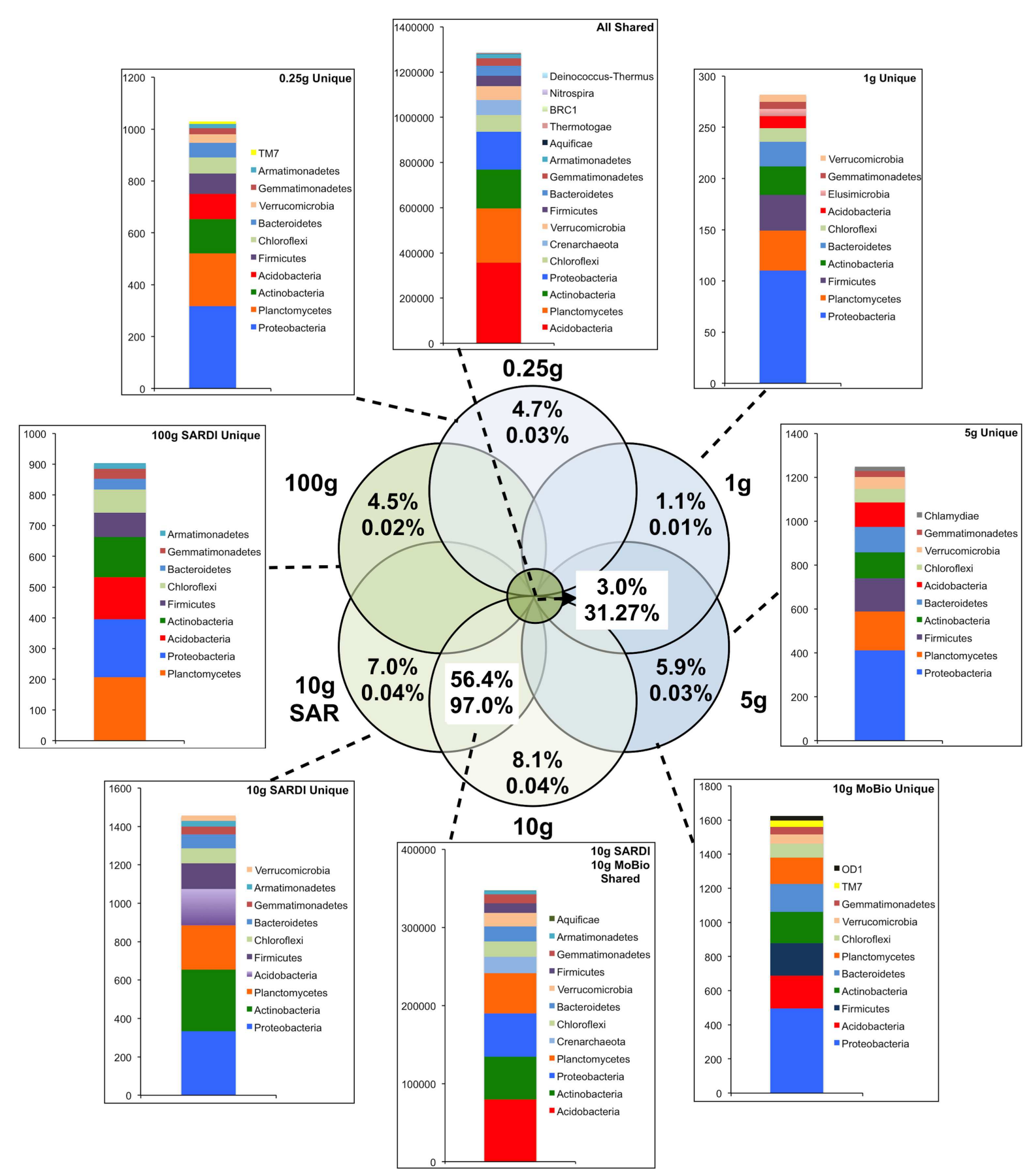

FIGURE 2 | Sequence abundances of the top most abundant unique OTUs for each sample size and their corresponding classification for the bacterial 16S rRNA gene sequences. OTUs that were shared among all sample sizes are shown in the "All Shared" inset with those shared between the SARDI $10 \mathrm{~g}$ and $\mathrm{MoBIO} 10 \mathrm{~g}$ in an additional inset. Within the Venn diagram, top numbers indicate the percent of total OTUs while the bottom represents the percent of total sequences.

the $10 \mathrm{~g}$ MoBIO extraction for the fungal community, but did not differ significantly for the bacterial community data (Table 1). The OTU data showed that only the total fungal OTUs were different between the two methods (Table 3). While the replicate dispersion between the two extractions methods for the $10 \mathrm{~g}$ samples were similar (Table 2) for both the fungal and bacterial communities in the two extractions, they were distinctly separated from each other in NMDS ordinations 


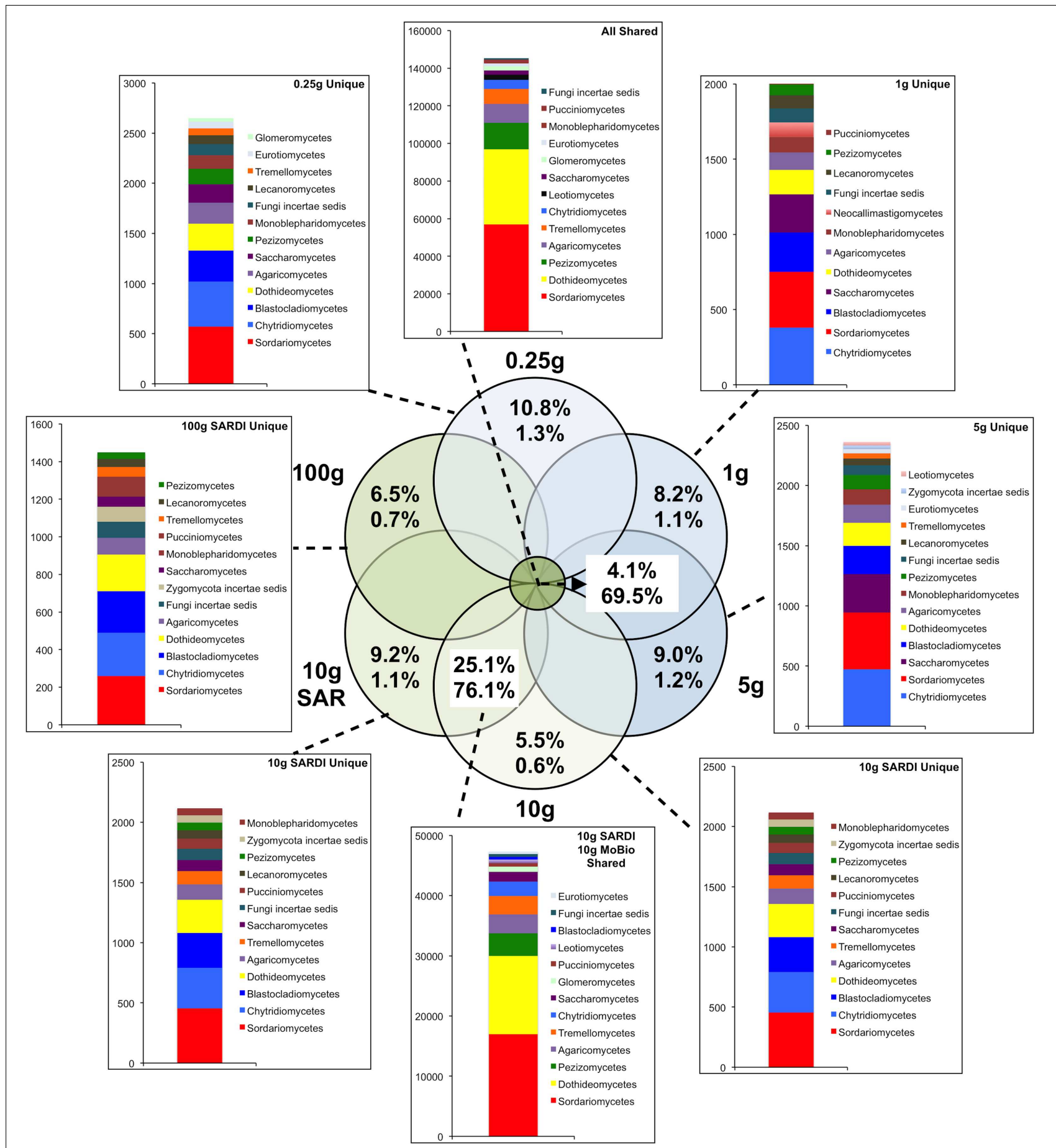

FIGURE 3 | Sequence abundances of the top most abundant unique OTUs for each sample size and their corresponding classification for the fungal $28 \mathrm{~S}$ gene sequences. OTUs that were shared among all sample sizes are shown in the "All Shared" inset with those shared between the SARDI $10 \mathrm{~g}$ and MoBIO $10 \mathrm{~g}$ in an additional inset. Within the Venn diagram, top numbers indicate the percent of total OTUs while the bottom represents the percent of total sequences.

(Figure 4). The extraction method differences illustrated in the fungal and bacterial community ordinations are also apparent in cluster analyses as the SARDI extractions cluster independently from most other samples (Supplementary Figures S2A,B in
Supplementary Data). In total, the $10 \mathrm{~g}$ SARDI and $10 \mathrm{~g}$ MoBIO extractions shared $97.0 \%$ of bacterial and $76.1 \%$ of fungal sequences contained within 56.4 and $25.1 \%$ of the total OTUs, respectively. 


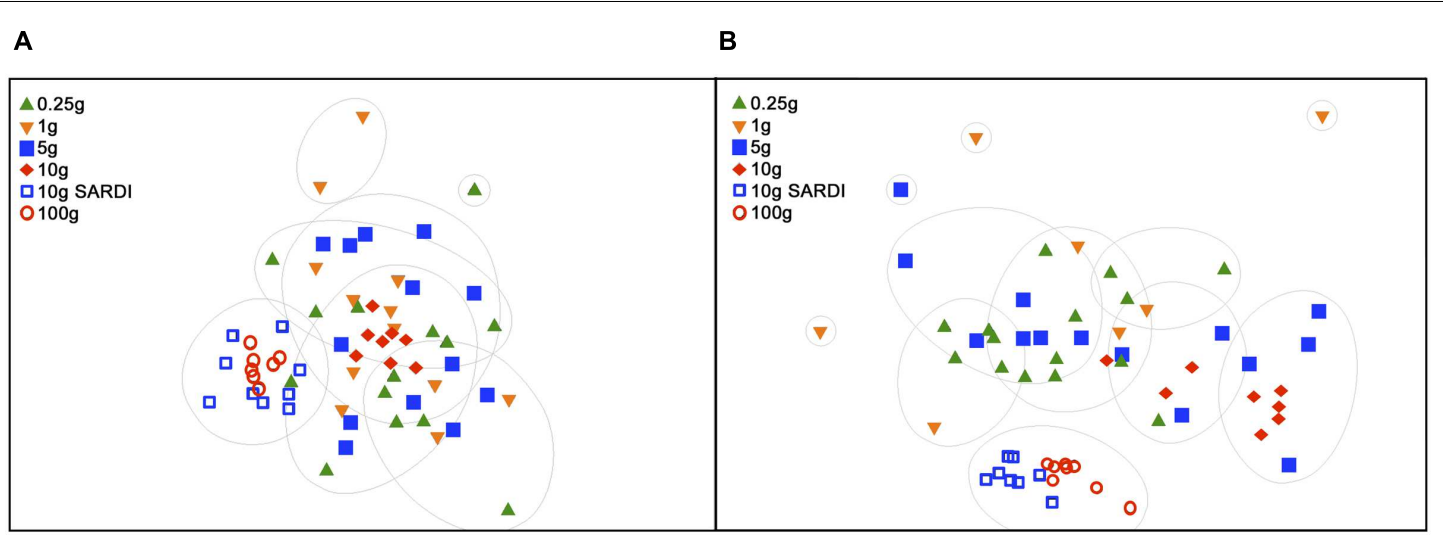

FIGURE 4 | Non-metric dimensional scaling (NMDS) of 28S rRNA gene data (A) and of 16S rRNA gene data (B). Groupings are based on SIMPROF with complete linkage clustering at 95\% confidence at 30\% (28S) and 60\% (16S) similarities. 2D stress was 0.21 (A) and 0.14 (B).

\section{OTU Abundance Contrasts}

Comparison of the number of OTUs to the number of sequences clearly indicates that the gain in the number of OTUs decreases with increasing sample size, such that they become almost all singletons at $100 \mathrm{~g}$. The mean OTU abundances for each sample size group were plotted pairwise to identify significant differences in relative abundances for specific taxa. For the bacterial community (Supplementary Figures S3A-E in Supplementary data), comparing $16 \mathrm{~S}$ rRNA gene OTU abundances of the $0.25 \mathrm{~g}$ extractions to all others reveals increasing variability as extraction size increases. This occurs particularly with the $16 \mathrm{~S}$ rRNA gene OTUs containing a greater number of sequences that may be important factors in discriminating samples or informing biological conclusions. For example, the comparison of $0.25 \mathrm{~g}$ with $100 \mathrm{~g}$ revealed that $75.0 \%$ of OTUs containing more than an average of 50 sequences among replicates were significantly different $(t$-test, $P<0.05)$ while only $16.4 \%$ of non-singleton or doubleton sequences containing less than 50 sequences were significantly different. The higher abundance OTUs were generally enriched in abundance in the larger sample size extractions with all pairwise comparisons. The taxonomic composition of these OTUs was varied and largely affiliated to Acidobacteria, unclassified Archaea and Rubrobacter. The $10 \mathrm{~g}$ and $100 \mathrm{~g}$ SARDI extractions that clustered closely in NMDS ordination exhibited a strong correlation in OTU abundances $\left(R^{2}=0.93, P<0.01\right)$. The weakest OTU abundance correlations were observed between the $10 \mathrm{~g}$ MoBIO and $10 \mathrm{~g}$ SARDI $\left(R^{2}=0.73, P<0.01\right)$ and $100 \mathrm{~g}$ SARDI $\left(R^{2}=0.61, P<0.01\right)$.

For the fungal data, the overall strength of the correlations using the mean $28 \mathrm{~S}$ gene OTU abundances among the extractions were weaker than that observed with the 16S rRNA gene data (Supplementary Figures S4A-E in Supplementary data). Correlations of the $0.25 \mathrm{~g}$ sample OTU abundances were fairly consistent when compared to the $1 \mathrm{~g}, 5 \mathrm{~g}$, and $10 \mathrm{~g}$ MoBio extractions $\left(R^{2}=0.61,0.64,0.61\right.$, respectively), but decreased largely with the comparisons to the SARDI $10 \mathrm{~g}\left(R^{2}=0.33\right)$ and $100 \mathrm{~g}$ extractions $\left(R^{2}=0.25\right)$. Again, the strongest correlation occurred between the two SARDI extractions $\left(R^{2}=0.83\right.$,
$P<0.01)$ while the weakest were found in all comparisons against the $100 \mathrm{~g}$ dataset $\left(R^{2}=0.25\right.$ to 0.35$)$. Comparing the $0.25 \mathrm{~g}$ with $100 \mathrm{~g}$ showed that $75.9 \%$ of OTUs containing more than an average of 20 sequences among replicates were significantly different between extraction sizes $(t$-test, $P<0.05)$ and were constrained to a few orders: $67 \%$ belonged to the order Pleosporales, $14 \%$ to Hypocreales, $14 \%$ to Sordariales, and 5\% to Tremellales, with classification bootstrap values ranging $70-100 \%$ at the genus level. In contrast, only $38.5 \%$ of non-singleton or doubleton were significantly different $(t$-test, $P<0.05)$.

\section{DISCUSSION}

\section{Comparison of Sample Sizes}

Based on 16S and 28S rRNA gene sequence analysis, sample size significantly influenced the overall bacterial and fungal community structure as measured by richness, evenness, diversity, and the dispersion among replicates. The largest richness, evenness, and diversity values were associated with the $10 \mathrm{~g}$ MoBIO extractions indicating that this soil sample extraction size range is optimal for soil community diversity assessments in this soil type. The dispersion metrics MVDISP, PERMDISP and within-group-average PERMANOVA similarities also show that, among the MoBIO extractions, the $10 \mathrm{~g}$ samples exhibited the lowest replicate variability. Sub-replicate dispersions were lower than the dispersion observed among all replicates within a particular extraction size, indicating that spatially close samples were more similar than those from another sub-plot within the same sampled area. These values also decreased with increasing sample size, reflecting the importance of larger extraction sizes even at smaller spatial scales, presumably due to small-scale spatial heterogeneity (microsites). Due to this lower dispersion and in the context of an experimental framework where treatment differences in both soil bacterial and fungal community structure are assessed, the $10 \mathrm{~g} \mathrm{MoBIO}$ extraction should provide a higher probability of detecting differences, though different soil types and/or niches (e.g., rhizosphere soil) may lead to different results. 
For the bacterial and fungal communities, the observation that the more abundant OTUs were more likely to exhibit a significant difference among extraction sizes indicates patchiness in the densities of the dominant taxa. These abundant OTUs appear to be located in "hot spots" within the soil; the smaller extraction sizes access the locally abundant but spatially rare. The correlation plots suggest that the larger samples (e.g., $10 \mathrm{~g}$ MoBIO) revealed both the locally abundant/spatially rare and the locally rare/spatially abundant bacterial and fungal OTUs. Rarefaction curves support this interpretation by showing a smaller degree of coverage, especially for the fungi. This patchiness or spatial clustering is often associated with fungi (Horton and Bruns, 2001), due to their association with decomposing organic residues and localized spores/resting structures. Indeed, the smaller proportion of fungal shared sequences contained within half the proportion of OTUs, compared to the bacteria, in the $0.25 \mathrm{~g}-10 \mathrm{~g}$ MoBio comparison further illustrate this fungal spatial patchiness at a small scale. It has been suggested that the detection of low abundance taxa such as the plant pathogenic fungi such as Cochliobolus sativus may be favoured by the larger sample size for extraction (Song et al., 2015). Also, a larger sample size will assist in reducing errors in predicting risk categories for soilborne fungal and nematode diseases based on pathogen inoculum measures from large fields (Ophel-Keller et al., 2008).

We argue that the conclusions on sample size presented by Ranjard et al. (2003) and discussed in a review by Lombard et al. (2011) are not wholly applicable to high throughput sequencing. Specifically, that the use of large soil samples are suitable for the description of the overall soil community structure while large numbers of small samples are more appropriate for a determination of local microbial diversity. Earlier sample size studies were based on less robust techniques such as ARISA (Ranjard et al., 2003) or DGGE (Ellinsøe and Johnsen, 2002; Kang and Mills, 2006) are limited in their ability to assess the 'rare biosphere' (Sogin et al., 2006). While dominant members may mask the signatures of minority populations using these techniques, our results suggest that sufficiently deep amplicon sequencing overcomes these limitations by revealing minority populations in the context of the overall community structure. This is supported by the higher diversity indices and larger number of non-singleton/doubleton OTUs in the $10 \mathrm{~g}$ sample. Moreover, if dominant populations did indeed mask the more rare OTUs then we would expect more unique OTUs in the $0.25 \mathrm{~g}$ sample compared to the $10 \mathrm{~g}$ sample. However, we found the opposite through presence/absence analyses; 1191 and 2414 unique fungal OTUs and 1074 and 1657 unique bacterial OTUs in the 0.25 and $10 \mathrm{~g}$ samples, respectively. Thus, these data suggest that larger soil samples should not directly bias against identification of new bacterial strains.

\section{Comparison of Extraction Methods}

The comparison of extraction methods using the $10 \mathrm{~g}$ MoBio and $10 \mathrm{~g}$ SARDI soil extractions showed that while fungal community richness, evenness and diversity was significantly greater in the MoBIO extraction, these differences were not significant in the bacterial community. This is despite the finding that the SARDI extraction recovered significantly more total fungal OTUs. The number of shared sequences between the $10 \mathrm{~g}$ MoBIO and $10 \mathrm{~g}$ SARDI for both bacteria (97.0\%) and fungi (76.1\%) suggest that the extraction methods are most comparable for bacterial community analyses.

Indeed, the number of shared sequences between extraction methods was similar to those found between the 0.25 and $10 \mathrm{~g}$ MoBio extractions (98.0\%-bacteria, 83.8\%-fungi), indicating an overall influence of extraction method. The sample dispersion and within group similarities indicate that both methods yielded equally reproducible replicate results. The interaction between the number of OTUs recovered and diversity measures is reflected in the discrepancy of the extraction method that recovered the most fungal OTUs in both the complete data and the non-singleton-doubleton (non-S-D) data. While SARDI recovered the most total fungal OTUs, a larger proportion of these OTUs were rare, leading to a low number of non-S-D OTUs. In this non-S-D data, the number of OTUs recovered from the $1 \mathrm{~g}, 5 \mathrm{~g}$, and $10 \mathrm{~g}$ MoBio extractions were similar.

The taxonomic composition of the bacterial OTUs showed that no specific lineages exhibited large differences in relative abundances (or presence/absence in the more abundant OTUs) among the different size MoBIO and SARDI extractions. In contrast, there were some differences observed among specific fungal lineages. The $0.25 \mathrm{~g}$ extractions had higher abundance OTUs containing unclassified Eukaryota Incertae sedis, unclassified Glomeromycota and Agaricomycetes. In contrast, both SARDI extractions (10 g and $100 \mathrm{~g}$ ) resulted in higher abundances of OTUs classified as Alternaria, unclassified Pleosporales and Cercophora. Members of the Pleosporales group, including Alternaria spp. and Cercophora spp., produce thick-walled, melanized spores. The recovery of DNA from environmental samples requires efficient cell lysis, especially from spores and other microbial resting structures. The SARDI DNA extraction method was originally standardized/calibrated to extract DNA from resting structures such as nematode cysts and fungal spores from soil samples (Ophel-Keller et al., 2008). The bead-beating intensity in the MoBIO protocol may not be as efficient in lysing these structures, resulting in lower relative abundances. Overall, these differences resulted in the low correlation between the MoBio and SARDI extractions. While the bead-beating SARDI and MoBIO PowerSoil ${ }^{\circledR}$ methods have been previously shown to result in very similar plant root DNA extraction efficiencies, as assessed by quantitative PCR (Haling et al., 2011), the aforementioned differences in OTU abundances associated with each extraction method, especially for the fungal data, would likely lead to differing biological conclusions, especially where explicit taxonomic associations are made.

\section{CONCLUSION}

In this study we found that soil sample size still plays a role in these rather homogenous soils that were collected during the non-crop season, without the rhizospheric influence of the growing crop, with uniform physical and chemical 
attributes, management histories, and aboveground crop responses. Nonetheless, these soil cores (and soil subsamples) still represented a range of microhabitats, including the crop residue detritusphere, the rhizospheric legacy from previous crops, the aggregatosphere and other microsites providing unique microbial habitats (Beare et al., 1995). Hence, the larger samples that encompassed a more even distribution of these habitats (microsites) revealed higher microbial diversity with lower replicate variation. However, these results may not apply to other, more specific microbial habitats or other soil types, especially soils with large aggregate structures. For example, the plant rhizosphere microbial community is considered to be more uniform in distribution (Hinsinger et al., 2009). In this circumstance, a smaller soil sample size may be adequate to cover the lower heterogeneity but also necessary to target this smaller habitat. In addition, highly structured soils would likely require soil screening in order to improve homogenization for DNA extraction.

In all, both sample size and extraction method significantly impacted fungal and bacterial community compositions as revealed by high throughput sequencing. This illustrates the essential requirement for transparency and consistency in extraction methods when comparing studies. While the $10 \mathrm{~g}$ sample reveals higher diversity, less dispersion among replicates, and more depth of taxa information, the value of the resolution gained needs to be considered relative to (1) the variation of the features/attributes of the system under study, (2) the resolution needed to answer the question and (3) extraction costs.

\section{REFERENCES}

Anderson, M. J. (2001). A new method for non-parametric multivariate analysis of variance. Austral Ecol. 26, 32-46. doi: 10.1111/j.1442-9993.2001.01070.pp.x

Anderson, M. J., Ellingsen, K. E., and McArdle, B. H. (2006). Multivariate dispersion as a measure of beta diversity. Ecol. Lett. 9, 683-693. doi: 10.1111/j.1461-0248.2006.00926.x

Bailey, V. L., McCue, L. A., Fansler, S. J., Boyanov, M. I., DeCarlo, F., Kemner, K. M., et al. (2013). Micrometer-sclale physical structure and microbial composition of soil macroaggregates. Soil Biol. Biochem. 65, 60-68. doi: 10.1016/j.soilbio.2013.02.005

Beare, M. H., Coleman, D. C., Crossley, D. A. Jr., Hendrix, P. F., and Odum, E. P. (1995). A hierarchical approach to evaluating the significance of soil biodiversity to biogeochemical cycling. Plant Soil 170, 5-22. doi: 10.1007/BF02183051

Bithell, S. L., Butler, R. C., McKay, A. C., and Cromey, M. G. (2013). Influences of crop sequence, rainfall and irrigation on relationships between Gaeumannomyces graminis var. tritici and take-all in New Zealand wheat fields. Australas. Plant Pathol. 42, 205-217. doi: 10.1007/s13313-0120168-9

Clarke, K. R. (1993). Non-parametric multivariate analyses of changes in community structure. Austral Ecol. 18, 117-143. doi: 10.1111/j.14429993.1993.tb00438.x

Clarke, K. R., and Gorley, R. N. (2006). PRIMER v6: User Manual/Tutorial. Plymouth: PRIMER-E.

Clarke, R. K., Somerfield, P. J., and Gorley, R. N. (2008). Testing of null hypotheses in exploratory community analyses: similarity profiles and biota-environment linkage. J. Exp. Mar. Biol. Ecol. 366, 57-69. doi: 10.1016/j.jembe.2008. 07.009

Cole, J. R., Wang, Q., Fish, J. A., Chai, B., McGarrell, D. M., Sun, Y., et al. (2014). Ribosomal database project: data and tools for high throughput rRNA analysis. Nucleic Acids Res. 42, D633-D642. doi: 10.1093/nar/gkt1244

\section{AUTHOR CONTRIBUTIONS}

Study design was performed by JT, VG, and RP. Field sampling was carried out by VG. Lab work and data collection was performed by RP and JY. Data interpretation was performed by RP, VG, JT, and JY. All authors contributed to manuscript preparation.

\section{ACKNOWLEDGMENTS}

This work was supported in part by Michigan State University through computational resources provided by the Institute for Cyber-enabled Research, and DOE grant DE-SC0004601. Funding support for VG was provided by the Grains RDC Soil Biology Initiative, Australia. This work was also stimulated by and disseminated through NSF Research Coordination Network Grant, RCN 1051481 and a CSIRO McMaster Fellowship for JT. Authors express their appreciation to Robin Manley, farmer and owner of the property at Avon, for allowing soil sampling.

\section{SUPPLEMENTARY MATERIAL}

The Supplementary Material for this article can be found online at: http://journal.frontiersin.org/article/10.3389/fmicb. 2016.00824

Edgar, R. C., Haas, B. J., Clemente, J. C., Quince, C., and Knight, R. (2011). UCHIME improves sensitivity and speed of chimera detection. Bioinformatics 27, 2194-2200. doi: 10.1093/bioinformatics/btr381

Ellinsøe, P., and Johnsen, K. (2002). Influence of soil sample size on the assessment of bacterial community structure. Soil Biol. Biochem. 34, 1701-1707. doi: 10.1016/S0038-0717(02)00156-6

Fish, J. A., Chai, B., Wang, Q., Sun, Y., Brown, C. T., Tiedje, J. M., et al. (2013). FunGene: the functional gene pipeline and repository. Front. Microbiol. 4:291. doi: 10.3389/fmicb.2013.00291

Gupta, V. V. S. R., and Germida, J. J. (2015). Soil aggregation: influence on microbial biomass and implications for biological processes. Soil Biol. Biochem. 80, A3-A9. doi: 10.1016/j.soilbio.2014.09.002

Haling, R. E., Simpson, R. J., McKay, A. C., Hartley, D., Lambers, H., OphelKeller, K., et al. (2011). Direct measurement of roots in soil for single and mixed species using a quantitative DNA-based method. Plant Soil 348, 123-137. doi: 10.1007/s11104-011-0846-3

Hattori, T. (1988). Soil aggregates as microhabitats of microorganisms. Rep. Inst. Agric. Tohoku Univ. 37, 23-36.

Hinsinger, P., Glen Bengough, A., Vetterlein, D., and Young, I. M. (2009). Rhizosphere: biophysics, biogeochemistry and ecological relevance. Plant Soil 321, 117-152. doi: 10.1007/s11104-008-9885-9

Horton, T. R., and Bruns, T. D. (2001). The molecular revolution in ectomycorrhizal ecology: peeking into the black box. Mol. Ecol. 10, 1855-1871. doi: 10.1046/j.0962-1083.2001.01333.x

Huang, C. Y., Kuchel, H., Edwards, J., Hall, S., Parent, B., Eckermann, P., et al. (2013). A DNA-based method for studying root responses to drought in field-grown wheat genotypes. Sci. Rep. 3:3194. doi: 10.1038/srep03194

Kang, S., and Mills, A. L. (2006). The effect of sample size in studies of soil microbial community structure. J. Microbiol. Methods 66, 242-250. doi: 10.1016/j.mimet.2005.11.013

Kozich, J. J., Westcott, S. L., Baxter, N. T., Highlander, S. K., and Schloss, P. D. (2013). Development of a dual-index sequencing strategy and curation pipeline 
for analyzing amplicon sequence data on the MiSeq Illumina sequencing platform. Appl. Environ. Microbiol. 79, 5112-5120. doi: 10.1128/AEM.01043-13

Liu, K.-L., Porras-Alfaro, A., Kuske, C. R., Elchorst, S. A., and Xie, G. (2012). Accurate, rapid taxonomic classification of fungal large-subunit rRNA genes. Appl. Environ. Microbiol. 78, 1523-1533. doi: 10.1128/AEM.06826-11

Lombard, N., Prestat, E., van Elsas, J. D., and Simonet, P. (2011). Soilspecific limitations for access and analysis of soil microbial communities by metagenomics. FEMS Microbiol. Ecol. 78, 31-49. doi: 10.1111/j.15746941.2011.01140.x

Nicol, G. W., Glover, L. A., and Prosser, J. I. (2003). Spatial analysis of archaeal community structure in grassland soil. Appl. Environ. Microbiol. 69, 7420-7428. doi: 10.1128/AEM.69.12.7420-7429.2003

Northcote, K. H., Hubble, G. D., Isbell, R. F., Thompson, C. H., and Bettenay, E. (1975). A Description of Australian Soils. East Melbourne, VIC: CSIRO.

Ophel-Keller, K., McKay, A., Hartley, D., Curran, H., and Curran, J. (2008). Development of a routine DNA-based testing service for soilborne diseases in Australia. Australas. Plant Pathol. 37, 243-253. doi: 10.1371/journal.pone.0082841

Penton, C. R., Gupta, V. V. S. R., Tiedje, J. M., Neate, S. M., Ophel-Keller, K., Gillings, M., et al. (2014). Fungal community structure in disease suppressive soils assessed by $28 \mathrm{~S}$ LSU gene sequencing. PLOS ONE 9:e93893. doi: 10.1371/journal.pone.0093893

Penton, C. R., St. Louis, D., Cole, J. R., Luo, Y., Wu, L., Schuur, E. A. G., et al. (2013). Fungal diversity in permafrost and tallgrass prairie soils under experimental warming conditions. Appl. Environ. Microbiol. 79, 7063-7072. doi: 10.1128/AEM.01702-13

Rainey, P. B., and Travisano, M. (1998). Adaptive radiation in a heterogeneous environment. Nature 3946, 9-72.

Ranjard, L., Lejon, D. P. H., Mougel, C., Schehrer, L., Merdinoglu, D., and Chaussod, R. (2003). Sampling strategy in molecular microbial ecology: influence of soil sample size on DNA fingerprinting analyses of fungal and bacterial communities. Environ. Microbiol. 5, 1111-1120. doi: 10.1046/j.14622920.2003.00521.x

Schloss, P. D., Westcott, S. L., Ryabin, T., Hall, J. R., Hartmann, M., Hollister, E. B., et al. (2009). Introducing mothur: open-source, platformindependent, community-supported software for describing and comparing microbial communities. Appl. Environ. Microbiol. 5, 7537-7541. doi: 10.1128/AEM.01541-09

Simpson, R. J., Richardson, A. E., Riley, I. T., McKay, A. C., Ballard, R. A., Ophel-Keller, K., et al. (2011). Damage to roots of Trifolium subterraneum L. (subterranean clover), failure of seedlings to establish and the presence of root pathogens during autumn-winter. Grass Forage Sci. 66, 585-605. doi: 10.1111/j.1365-2494.2011.00822.x

Sogin, M. L., Morrison, H. G., Huber, J. A., Welch, D. M., Huse, S. M., Neal, P. R., et al. (2006). Microbial diversity in the deep sea and the underexplored "rare biosphere." Proc. Natl. Acad. Sci. U.S.A. 103, 12115-12120. doi: 10.1073/pnas.0605127103

Song, Z., Schlatter, D., Kennedy, P., Kinkel, L. L., Kistler, H. C., Nguyen, N., et al. (2015). Effort versus reward: preparing samples for fungal community characterization in high-throughput sequencing surveys of soils. PLoS ONE 10:e0127234. doi: 10.371/journal.pone.0127234

Stres, B., Mahne, I., Avgustin, G., and Tiedje, J. M. (2004). Nitrous oxide reductase (nosZ) gene fragments differ between native and cultivated Michigan soils. Appl. Environ. Microbiol. 70, 301-309. doi: 10.1128/AEM.70.1.301309.2004

Tiedje, J. M., Asuming-Brempong, S., Nüsslein, K., Marsh, T. L., and Flynn, S. J. (1999). Opening the black box of soil microbial diversity. Appl. Soil Ecol. 13, 109-122. doi: 10.1016/S0929-1393(99)00026-8

Vos, M., Wolf, A. B., Jennings, S. J., and Kowalchuk, G. A. (2013). Micro-scale determinants of bacterial diversity in soil. FEMS Microbiol. Rev. 37, 936-954. doi: $10.1111 / 1574-6976.12023$

Conflict of Interest Statement: The authors declare that the research was conducted in the absence of any commercial or financial relationships that could be construed as a potential conflict of interest.

Copyright (c) 2016 Penton, Gupta, Yu and Tiedje. This is an open-access article distributed under the terms of the Creative Commons Attribution License (CC BY). The use, distribution or reproduction in other forums is permitted, provided the original author(s) or licensor are credited and that the original publication in this journal is cited, in accordance with accepted academic practice. No use, distribution or reproduction is permitted which does not comply with these terms. 